\title{
Intramural duodenal hematoma as a complication of pancreatic cancer
}

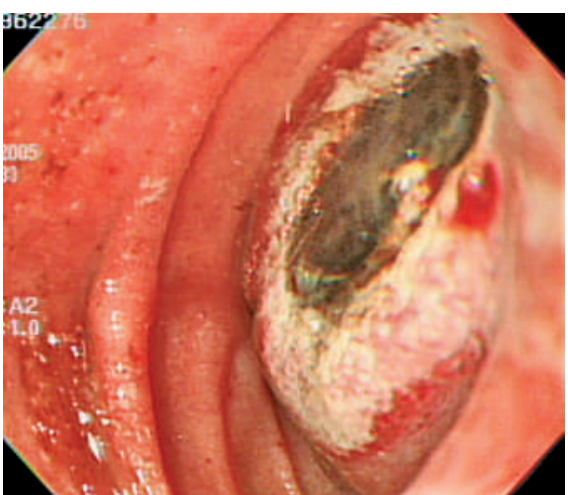

Figure 1 Endoscopic view of the duodenal bulb shows a volcanic ulcerative mass that almost completely obstructs the lumen.

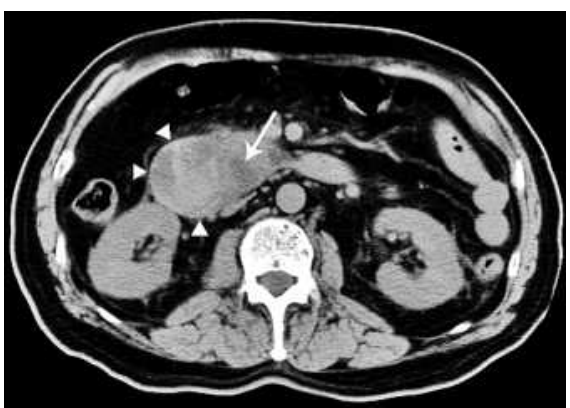

Figure 2 Unenhanced abdominal computed tomography scan shows a heterogeneous, hyperdense mass in the right anterior pararenal space adjacent to the duodenum (arrowheads). A low-attenuation mass is shown in the uncinate process of the pancreas (arrow).

Intramural duodenal hematoma (IDH) occurs mainly in children, secondarily to blunt abdominal injury. In adults, blunt abdominal trauma accounts for $70 \%$ of IDH cases [1]. Nontraumatic causes of IDH in adults include coagulation disorders, duodenal biopsy, and endoscopic injection therapy for bleeding ulcers [2]. Very few cases of IDH of pancreatic origin have been reported [3-5]. We describe a case of IDH presenting as a complication of pancreatic cancer. This report highlights the need to consider a malignant process as a possible etiology of IDH.

A 70-year-old man was admitted to hospital with coffee ground emesis and epigastralgia. There was no history of alcohol use, anticoagulant therapy, nonsteroidal anti-inflammatory drug (NSAID) use, or

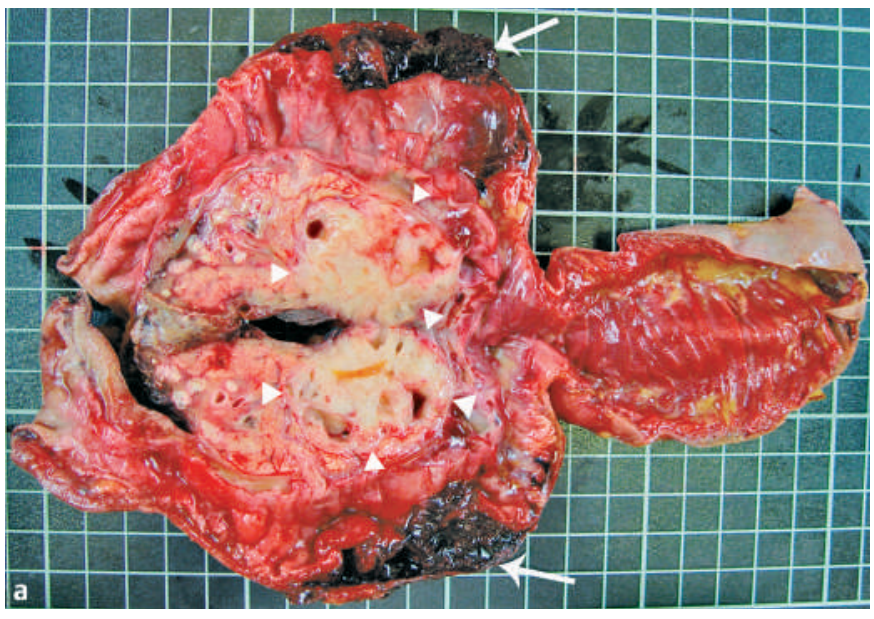

Figure 3 Gross appearance of the resected specimen. a The hematoma is visible within the wall of the duodenum (arrows), and an ovoid tumor is present in the uncinate process of the pancreas (arrowheads). $\mathbf{b}$ The tumor is seen invading to the wall of the duodenum (arrow).

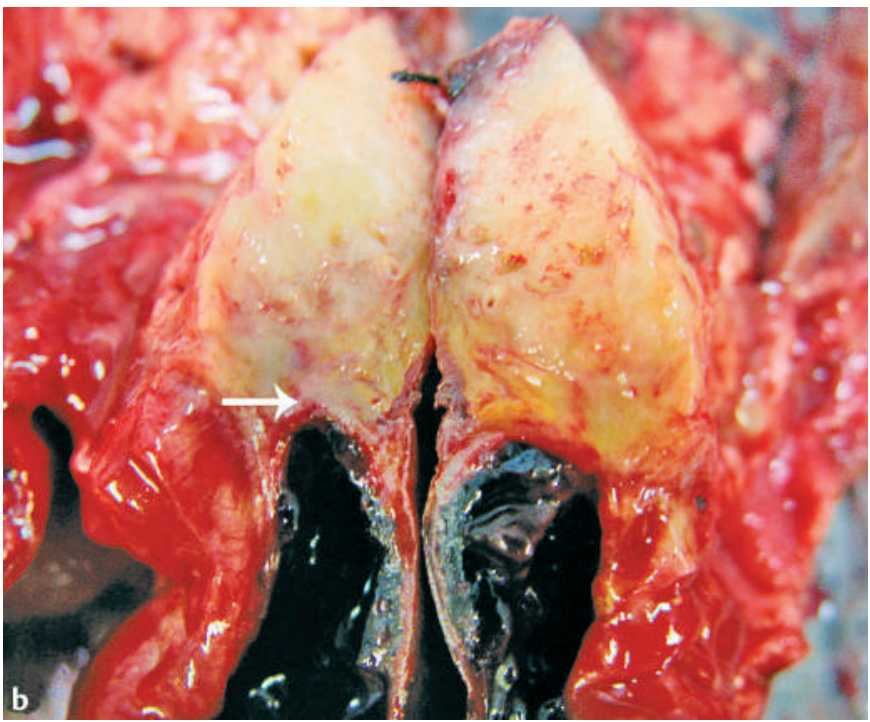

recent abdominal trauma. On esophagogastroduodenoscopy, multiple distal esophageal ulcers and a volcanic ulcerative mass in the duodenal bulb, which had a significantly narrowed lumen, were noted (Figure 1). Biopsy specimens of the mass consisted of duodenal mucosal tissue with ulceration that was infiltrated by inflammatory cells but not tumor cells. Abdominal computed tomography demonstrated a heterogeneous, hyperdense mass located in the right anterior pararenal space and a low-attenuation mass in the uncinate process of the pancreas (Figure 2). A diagnosis of an intramural duodenal hematoma secondary to the pancreatic mass was made.
The patient underwent a Whipple procedure. An ovoid tumor, measuring $3 \times 3 \times 2.8 \mathrm{~cm}$, was located in the uncinate process of the pancreas (Figure 3 ). The duodenum was gray, fleshy, and soft, with an $8 \times 5 \times 1.8 \mathrm{~cm}$ hemorrhagic cystic lesion within the duodenal wall, which was in contact with the margin of the pancreatic tumor (Figure 3). On microscopy, moderately differentiated ductal adenocarcinoma of the uncinate process of the pancreas, with invasion to the duodenal submucosa, was seen. Postoperatively, the patient recovered uneventfully and was discharged after 15 days. 


\section{UCTN}

Endoscopy_UCTN_Code_TTT_1AO_2AB

Endoscopy_UCTN_Code_TTT_1AO_2AD

\section{A.-L. Chou, K.-C. Tseng, Y.-H. Hsieh, W.-F. Feng, C.-A. Tseng}

Division of Gastroenterology, Department of Internal Medicine, Buddhist Dalin Tzu Chi General Hospital, Chia-Yi, Taiwan.
References

${ }^{1}$ Jewett TC Jr, Caldarola V, Karp MP et al. Intramural hematoma of the duodenum. Arch Surg 1988; 123: 54-58

2 Rohrer B, Schreiner J, Lehnert P et al. Gastrointestinal intramural hematoma, a complication of endoscopic injection methods for bleeding peptic ulcers: a case series. Endoscopy 1994; 26: 617-621

${ }^{3}$ Fesenmyer ME, Nelson DB. Intramural duodenal hematoma due to pancreatitis. J Clin Gastroenterol 1998; 26: 350-352

${ }^{4}$ Bellens L, Van Hee R, Vanderstighelen Y, Vanderputte $S$. Intramural duodenal hematoma of pancreatic origin. Hepatogastroenterology 1999; 46: 930-932

${ }^{5}$ Van Spreeuwel JP, van Gorp LH, Bast TJ, Nadorp JH. Intramural hematoma of the duodenum in a patient with chronic pancreatitis. Endoscopy 1981; 13: 246-248
Corresponding author

\section{C.-A. Tseng, MD}

Division of Gastroenterology Department of Internal Medicine Buddhist Dalin Tzu Chi General Hospital 2 Min-Sheng Road, Dalin, Chia-Yi Taiwan

Fax: $\quad+886-5-2641607$

Email: a4867@ms14.hinet.net 